\title{
Encuesta nacional sobre dolor en las urgencias hospitalarias
}

F. Caba Barrientos 1 , M.C. Benito Alcalá ${ }^{2}$, A. Montes Pérez ${ }^{3}$, J.L. Aguilar Sánchez ${ }^{4}$, R. de la Torre Liébana ${ }^{5}$ y

C. Margarit Ferri'; Grupo de Trabajo de Dolor Agudo de la Sociedad Española de Dolor (SED)

Servicio de Anestesiología, Reanimación y Tratamiento del Dolor. ${ }^{1}$ Hospital Universitario de Valme. Sevilla. ${ }^{2}$ Hospital Universitario Gregorio Marañón. Madrid. ${ }^{3}$ Parc de Salut Mar. Barcelona. ${ }^{4}$ Hospital Son Llàtzer. Palma de Mallorca. ${ }^{5}$ Hospital Universitario Carlos Haya. Málaga. ${ }^{6}$ Hospital General de Alicante. Alicante

Caba Barrientos F, Benito Alcalá MC, Montes Pérez A, Aguilar Sánchez JL, de la Torre Liébana R, Margarit Ferri C. Encuesta nacional sobre dolor en las urgencias hospitalarias. Rev Soc Esp Dolor 2014; 21(1): 3-15.

\begin{abstract}
Objective: To determine the epidemiology and management of acute pain in the emergency services (ES) of Spanish hospitals.

Method: Survey "on line" to doctors responsible for the ES from a sample of different types of hospital across the country. Aspects of pain epidemiology, assessment, institutional resources, types of treatments and specific training aspects in pain practitioners in the field of ES are investigated.

Results: With a response rate of $37 \%$ is estimated that $42.8 \%$ of patients come to the ES with pain as the main reason. Musculoskeletal pain is the most represented and more intense. Is usually assessed by scales in $58.3 \%$ of hospitals, although only $23.9 \%$ have specific documentation to do so. Major opioids are used in $16.9 \%$ of patients with pain, weak opioids in $25.4 \%$ and acetaminofen/NSAID $72.3 \%$. Possess analgesic protocols refer $46.3 \%$, and acute pain services or institutional pain programs (APS-IPP) $13.4 \%$. Only $20.9 \%$ of the hospitals have specific training activity in this area. With few differences between them, hospitals with APS-IPP tend to evaluate and document the pain, using more opioids and protocols, while providing more training.

Conclusion: Pain is still present and is underevaluated in the ES. At the institutional and individual level there is ample room for improvement in this field must consider new strategies, attitudes and organizational solutions.
\end{abstract}

Financiación: Estudio financiado por la Sociedad Española de Dolor.

Recibido: $02-11-12$

Aceptado: 05-02-13
Key words: Emergency service. Pain management. Analgesics.

\section{RESUMEN}

Objetivo: Conocer aspectos epidemiológicos y del manejo del dolor agudo en los servicios de urgencia (SU) de los hospitales españoles.

Método: Encuesta on line a los responsables médicos de los SU de una muestra de diferentes tipos de hospitales de todo el territorio nacional. Se investigan aspectos relativos a la epidemiología del dolor, evaluación, recursos institucionales, tipos de tratamientos y aspectos formativos específicos en dolor de los profesionales en el ámbito de los SU.

Resultados: Con una tasa de respuesta del $37 \%$ se estima que el $42,8 \%$ de los pacientes acuden a los SU con dolor como principal motivo. El dolor músculo-esquelético es el más representado y de mayor intensidad. Se evalúa habitualmente mediante escalas en el 58,3\% de los hospitales, aunque sólo el 23,9 \% tienen documentación específica para hacerlo. Opioides mayores se utilizan en el 16,9\% de los pacientes con dolor, opioides débiles en el 25,4\% y paracetamol/AINE en el 72,3\%.

Refieren poseer protocolos analgésicos el 46,3\% y unidades de dolor agudo o programas institucionales de dolor (UDA-PID) el 13,4 \%. Sólo el 20,9\% de los hospitales realiza actividad formativa específica en este ámbito. Con escasas diferencias entre centros, los hospitales con UDA-PID tienden a evaluar y documentar más el dolor, utilizan más los opioides mayores, los protocolos y facilitan más la formación.

Conclusión: El dolor sigue estando presente y es infravalorado en los SU. A nivel institucional e individual existe un amplio margen de mejora en este campo que habrá de contemplar estrategias, actitudes y soluciones organizativas.

Palabras clave: Servicio de urgencia. Tratamiento del dolor. Analgésicos. 


\section{INTRODUCCIÓN}

El dolor es un síntoma muy prevalente en las instituciones sanitarias que se considera pobremente manejado en los servicios de urgencias (SU). Estudios realizados en hospitales norteamericanos identifican la oligoanalgesia como una constante instalada desde hace varias décadas en los emergency departments, donde el porcentaje de pacientes que experimenta dolor de intensidad grave puede ser tan alto como del $78 \%$ y los tratamientos analgésicos utilizados son escasamente efectivos (1-7). Igualmente, estudios recientes han venido a demostrar cómo Europa no es ajena a una realidad en la que el dolor no se evalúa lo suficiente, los analgésicos potentes son infrautilizados y el retardo en el tratamiento es común en estos servicios (8-11). Aunque el conocimiento y el análisis de este problema ha servido como punto de partida para la implementación de medidas útiles para la mejora en el manejo del dolor en esta particular área (12-16), al igual que ha ocurrido con el dolor agudo postoperatorio (17), estas medidas no se han generalizado ni han resuelto el problema.

En el año 2000 la Sociedad Española del Dolor (SED) hizo públicos los datos de un estudio clínico-epidemiológico sobre el dolor en los SU de 10 hospitales españoles de diferentes comunidades, que registró información sobre la asistencia a 3.585 pacientes, de los cuales el $63,9 \%$ presentaron dolor con una duración media de 5,5 horas cuando se trataba de un dolor agudo. La intensidad del dolor fue moderada en el $49 \%$, leve en el $26 \%$ y grave en el $25 \%$, siendo este último más prevalente en fin de semana (27,6 vs. $21,5 \%)$. El tiempo medio que tuvieron que esperar los pacientes para recibir analgesia fue de 43 minutos. El dolor agudo fue el más referido con el $96,4 \%$ de los casos y con una elevada representación del dolor relacionado con traumatismos, golpes o contusiones $(41,9 \%)$, mientras que en el $95,4 \%$ de la totalidad de los pacientes atendidos, el dolor formaba parte del motivo de acudir a urgencias (18).

La alta prevalencia y las deficiencias en el manejo del dolor músculo-esquelético en las urgencias de los hospitales españoles también han sido puestas de manifiesto en otros estudios, donde la infraestimación de su intensidad (19) y la incorrecta utilización de los recursos analgésicos (20), se han presentado como principales causas determinantes del inadecuado tratamiento.

En el año 2011, y con motivo del Año Internacional del Dolor Agudo declarado por la Asociación Internacional para el Estudio del Dolor (IASP), la SED en colaboración con la Sociedad Española de Medicina de Urgencias y Emergencias (SEMES) propusieron y coordinaron un estudio a nivel nacional que abordara el dolor en urgencias y que fue diseñado por el Grupo de Trabajo de Dolor Agudo de la SED. El principal objetivo considerado por este estudio fue describir aspectos relacionados con la epi- demiología y manejo del dolor en los SU en una muestra representativa de los hospitales españoles (excluida la urgencia obstétrica y pediátrica), a través de los datos proporcionados por la contestación on line de los responsables médicos a un cuestionario estructurado.

\section{MÉTODO}

A partir del Catálogo Nacional de Hospitales 2011, editado por el Ministerio de Sanidad, Política Social e Igualdad, y actualizado con fecha 31 de diciembre de 2010, se identificaron 302 hospitales con más de 150 camas y un servicio de urgencias, de los cuales 270 correspondían a hospitales generales de dependencia pública y 32 a hospitales concertados con el Sistema Nacional de Salud. Los hospitales fueron divididos según el número de camas en aquellos con más de 1.000 camas, los que tenían entre 400 y 1.000 , los que tenían entre 200 y 400 camas y aquellos con menos de 200. También fueron identificadas otras características de los centros como el promedio de admisiones/día en las urgencias y la impartición de formación postgrado, siendo considerada como tal aquella que contemplaba, al menos, la formación MIR en medicina de familia.

Un cuestionario estructurado con una encuesta on line fue enviado a los responsables médicos de los servicios de urgencias de una muestra de los centros de referencia, con un total de 179 hospitales seleccionados: el $100 \%$ de los centros de más de 1.000 camas, un $75 \%$ de los centros de 400 a 1.000 camas y el $50 \%$ de los centros de menos de 400 camas, donde estaban representadas todas las Comunidades Autónomas. La muestra de centros fue aleatoria y estratificada, con diferentes fracciones de muestreo según el tamaño del centro y lo bastante elevadas por estrato, como para garantizar una cobertura amplia y representativa.

El periodo para la recogida de información desde los centros participantes comenzó el 1 de junio de 2011 y se extendió cuarenta días hasta el 10 de julio. Durante este periodo se realizó un seguimiento continuo de las respuestas con dos recordatorios de participación para aquellos centros que no contestaron al primero.

Los aspectos a registrar sobre el dolor en urgencias y su manejo que el cuestionario contemplaba aludían a los siguientes datos (ver Anexo I para el detalle):

1. Prevalencia y epidemiología. Estimación de porcentaje de pacientes con dolor como principal motivo de consulta y su causa. Distribución según topografía e intensidad.

2. Evaluación. Documentación del dolor. Evaluación del resultado analgésico y dolor al alta.

3. Recursos institucionales. Presencia de programas institucionales de dolor (PID) o unidades de dolor agudo (UDA) con ámbito de actuación en el servicio de urgencias. Disponibilidad de registros y escalas específicas para el dolor. 
4. Tratamiento. Existencia de protocolos. Utilización y prioridades en el uso de los diferentes analgésicos, técnicas y vías de administración.

5. Formación. Existencia de programas de formación específica en dolor para el staff y personal eventual.

Con los datos de las respuestas que se esperaban obtener se pretendía fundamentalmente realizar un análisis descriptivo de la situación del dolor y su manejo en los servicios de urgencias españoles, más que contrastar hipótesis buscando algún tipo de inferencia estadística.

\section{RESULTADOS}

De los 179 hospitales encuestados se recibió contestación de 67 (tasa global de respuesta del 37 \%). La muestra la conformaron 9 hospitales con más de 1.000 camas (10\% de los hospitales respondedores), 23 entre 400 y 999 camas (31\%), 12 entre 200 y 399 camas ( $25 \%$ ) y con menos de 200 camas, 23 (34\%). Los hospitales con más de 400 camas supusieron el $45 \%$ de los respondedores. $\mathrm{La}$ distribución por CC. AA. que cumplimentaron la encuesta se representa en la figura 1.

En la tabla I se reflejan las características de los hospitales que contestaron la encuesta, la relación público-privado y la presencia de UDA o PID, así como si tenían o no formación universitaria.

El 80,6\% de los hospitales contestaron tener formación postgrado $(\mathrm{n}=54)$ y PID o UDA sólo lo refirieron el $13,4 \%$ $(\mathrm{n}=9)$, siendo más frecuentes estos dispositivos asistencia- les en los hospitales no universitarios y en los que atendían entre 200 y 400 pacientes/día (Fig. 2). Además, de estos 9 centros que declararon tener un PID o una UDA, en 4 de ellos algún personal del staff del SU formaba parte de su organización o tenía alguna responsabilidad, sin que entre estos hubiera ningún hospital universitario, ni con atención a más de 400 pacientes/día.

El porcentaje medio estimado de pacientes que acuden con dolor como único o principal motivo de admisión en urgencias fue del $42,8 \%$ sin diferencias ostensibles entre los distintos tipos de hospitales (Tabla II). Las principales causas relacionadas con el dolor fueron los traumatismos $(39,2 \%)$ y la agudización de un dolor crónico $(27,7 \%)$. Dolor de intensidad grave fue estimado en el $15,9 \%$ de los pacientes. Considerando los de mayor intensidad, el dolor músculo-esquelético también fue el más referido entre los tres más graves $(62,7 \%)$, seguido por el dolor abdominal $(52,2 \%)$ y de vía urinaria (50,7 \%) (Fig. 3).

Globalmente refieren disponer de protocolos para el tratamiento del dolor en el SU el 46,3\% de los responsables encuestados (77,8\% en los hospitales con PID o UDA frente al $41,4 \%$ en los que no la tienen). En cuanto al número de pacientes atendidos, los SU que más utilizan protocolos analgésicos son los que asisten entre 200 y 400 pacientes/ día $(60,9 \%)$, seguidos de los que atienden a más de 400 (45,5\%) y los menos de 200 (36,4\%).

Los antiinflamatorios no esteroideos y el paracetamol se utilizan en $72,3 \%$ de los pacientes con dolor, opioides débiles en el $25,4 \%$, mientras que los opioides mayores se utilizan en el 16,9\% y se estima una utilización de anes-

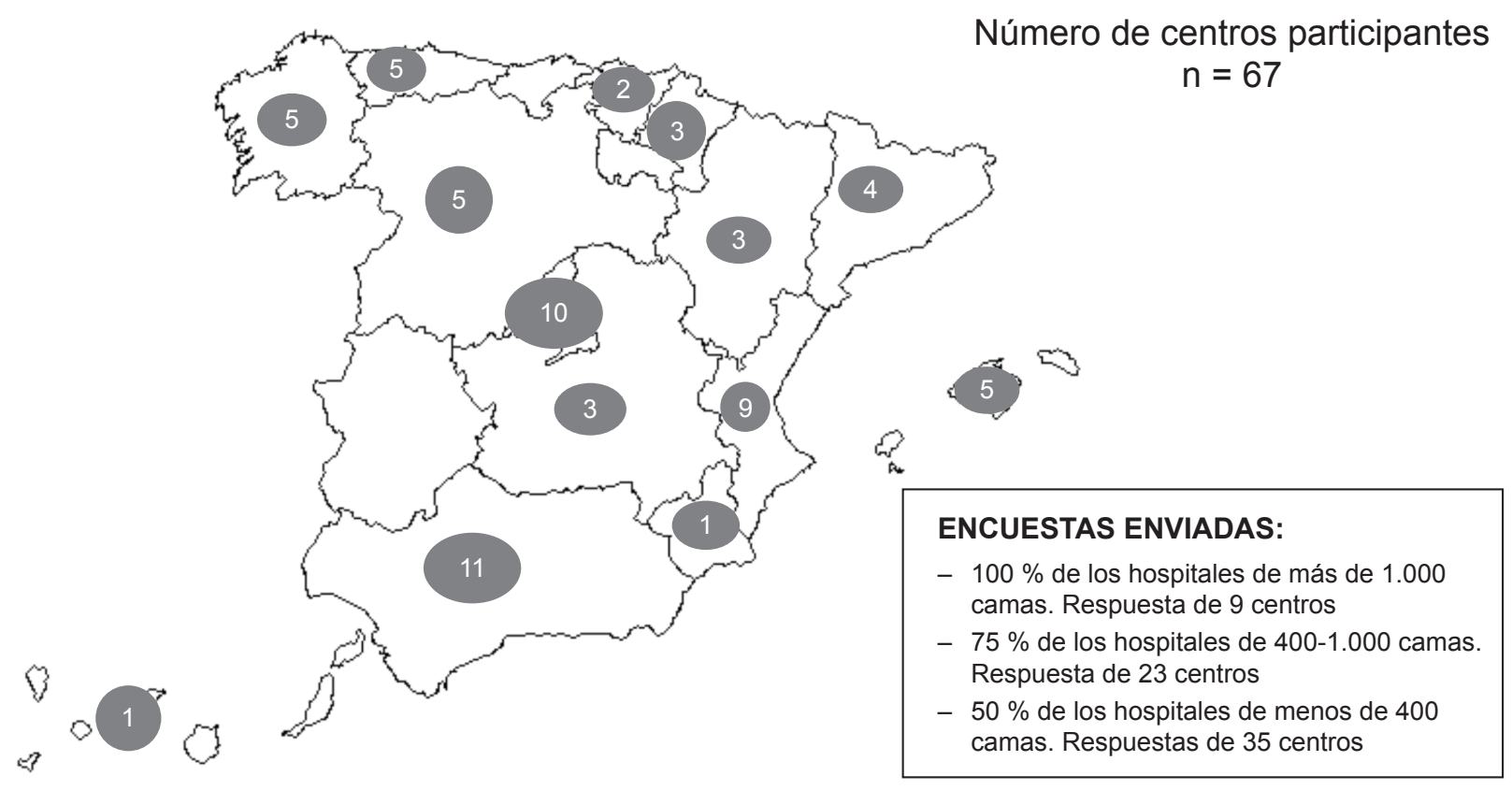

Fig. 1. Distribución geográfica de los 67 hospitales que respondieron la encuesta. 
TABLA I. CARACTERÍSTICAS DE LOS HOSPITALES QUE CONTESTARON LA ENCUESTA

\begin{tabular}{ccccccccc}
\hline & & \multicolumn{2}{c}{ Formación } & \multicolumn{2}{c}{ UDA o PID } & \multicolumn{3}{c}{ Demanda atendida } \\
\cline { 3 - 8 } & Total & $\begin{array}{c}\text { Universitario } \\
\text { y postgrado }\end{array}$ & $\begin{array}{c}\text { No } \\
\text { universitario }\end{array}$ & Si & No & $\begin{array}{c}\text { Menos de } \\
\text { 200 pac./día }\end{array}$ & $\begin{array}{c}\text { Entre 200- } \\
\text { 400 pac/día }\end{array}$ & $\begin{array}{c}\text { Más de 400 } \\
\text { pac/día }\end{array}$ \\
\hline Número & 67 & 54 & 13 & 9 & 58 & 33 & 23 & 11 \\
Público & $58(86,6)$ & $48(90,7)$ & $9(69,2)$ & $6(66,7)$ & $52(89,7)$ & $28(84,8)$ & $20(87,0)$ & $10(90,9)$ \\
Privado & $9(13,4)$ & $5(9,3)$ & $4(30,8)$ & $3(33,3)$ & $6(10,3)$ & $5(15,2)$ & $3(13,0)$ & $1(9,1)$ \\
\hline
\end{tabular}

Valores en número (y porcentaje) de hospitales. UDA: unidad de dolor agudo; PID: programa institucional de dolor.

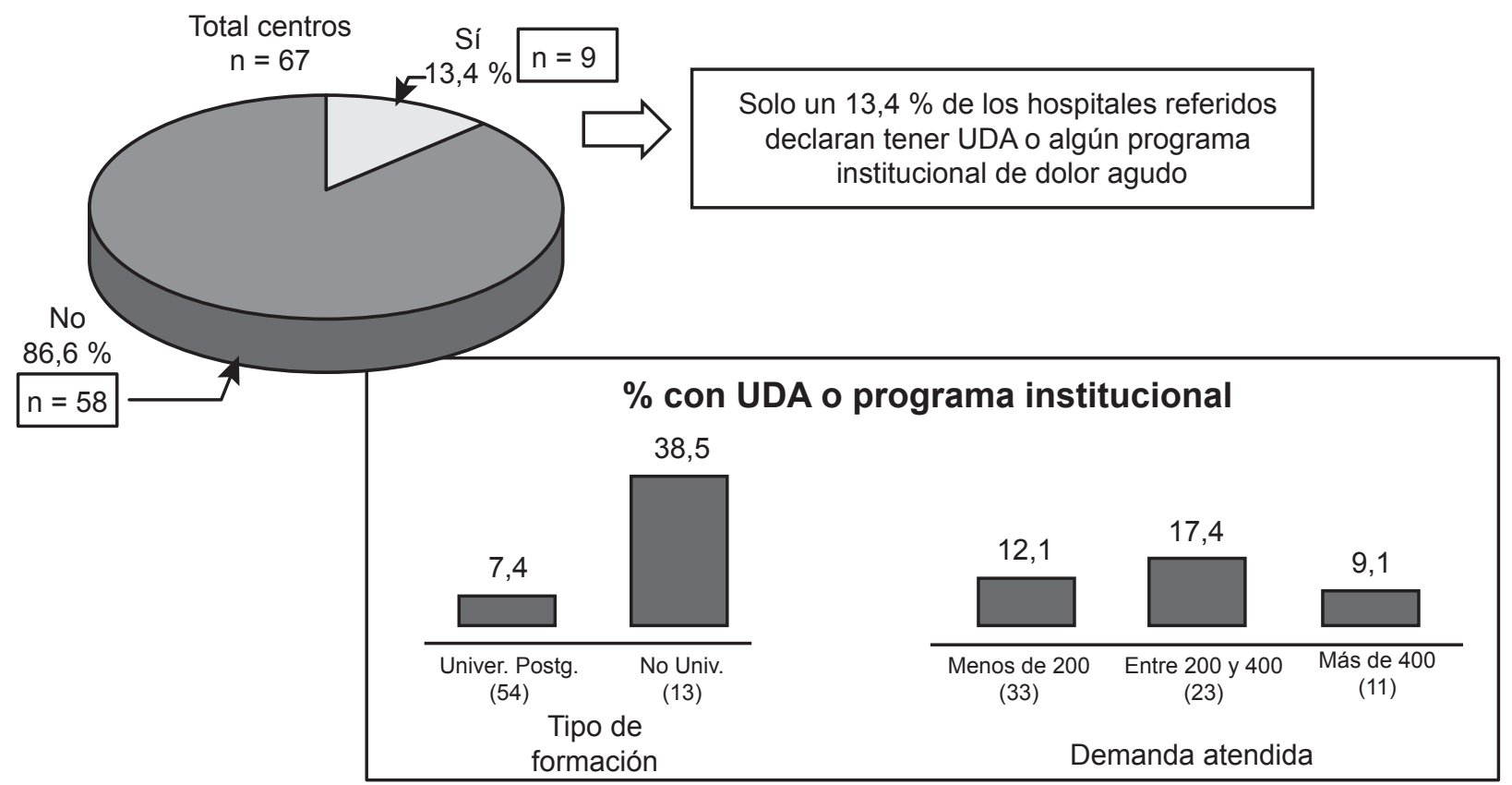

Fig. 2. Unidades del dolor agudo o programa institucional en los centros hospitalarios.

tésicos locales en el 8,4\%. Estos tratamientos analgésicos fueron similares en distintos tipos de hospitales (Tabla III), aunque hay una tendencia entre los hospitales grandes y los hospitales con PID-UDA a usar más los opioides mayores.

El tramadol fue el opioide más citado en primer lugar $(55,2 \%)$, seguido de la morfina $(22,4 \%)$, petidina $(11,9 \%)$ y fentanilo $(10,4 \%)$; pero cuando se computaron los tres opioides más usados en cada centro, la morfina se citó en el $86,6 \%$, seguida del tramadol $(80,6 \%)$, petidina $(73,1 \%)$, fentanilo $(53,7 \%)$ y codeína (4,5\%) (Fig. 4).

La vía intravenosa fue la referida como la más utilizada para la administración de opioides (91\%), seguida de la subcutánea $(22,4 \%)$, intramuscular $(6 \%)$ y oral $(6 \%)$.

Entre los no opioides el paracetamol fue el más utilizado (49,3\%), seguido del dexketoprofeno (20,9\%), metamizol
(19,4\%), ketorolaco (7,5\%), diclofenaco (1,5\%) e ibuprofeno $(1,5 \%)$; en cambio cuando se computaron los tres más utilizados en cada centro se citaron: metamizol (89,6 \%), paracetamol (82,1\%), dexketoprofeno $(62,7 \%)$, ketorolaco $(29,9 \%)$, ibuprofeno $(22,4 \%)$, diclofenaco $(13,4 \%)$; siendo la vía intravenosa la más utilizada también $(85,1 \%)$ frente a la intramuscular $(34,3 \%)$ y oral $(23,9 \%)$ (Fig. 4).

Se evalúa el dolor en urgencias con frecuencia, o sistemáticamente mediante alguna escala de dolor, en el 58,3\% de los hospitales. También se evalúa el resultado del tratamiento analgésico en el 64,2 \% y al alta en el 70,2 \% (Fig. 5).

Las escalas más utilizadas para registrar el dolor son la visual analógica (EVA) y la verbal numérica (ENV), seguida de la escala categórica, con diferencias importantes entre los hospitales con y $\sin$ PID-UDA (44,4 \% frente al 20,7 \%) (Tabla II). 
TABLA II. ASPECTOS RELACIONADOS CON LA VALORACIÓN Y REGISTRO DEL DOLOR, EN DIFERENTES TIPOS DE HOSPITALES

\begin{tabular}{|c|c|c|c|c|c|c|c|c|}
\hline & \multirow[b]{2}{*}{$\begin{array}{c}\text { Total } \\
(n=67)\end{array}$} & \multicolumn{2}{|c|}{ Formación } & \multicolumn{2}{|c|}{$U D A-P I D$} & \multicolumn{3}{|c|}{ Demanda atendida } \\
\hline & & $\begin{array}{c}\text { Universit. } \\
\text { postgrado } \\
(n=54)\end{array}$ & $\begin{array}{c}\text { No } \\
\text { universitario } \\
(n=13)\end{array}$ & $\begin{array}{c}S i \\
(n=9)\end{array}$ & $\begin{array}{c}N o \\
(n=58)\end{array}$ & $\begin{array}{c}\text { Menos de } \\
200 \text { pac./ } \\
\text { día } \\
(n=33)\end{array}$ & $\begin{array}{c}\text { Entre } 200- \\
400 \text { pac. } \\
\text { día } \\
(n=23)\end{array}$ & $\begin{array}{c}\text { Más de } \\
400 \text { pac./ } \\
\text { día } \\
(n=11)\end{array}$ \\
\hline $\begin{array}{l}\text { Dolor motivo de } \\
\text { admisión }\end{array}$ & 42,8 & 42,1 & 45,8 & 43,3 & 42,7 & 45 & 40,6 & 40,9 \\
\hline \multicolumn{9}{|l|}{ Escala utilizada } \\
\hline Catégorica & 15,9 & 15,7 & 16,7 & 33,3 & 13 & 12,5 & 19 & 20 \\
\hline EVN & 39,7 & 45,1 & 16,7 & 22,2 & 42,6 & 40,6 & 38 & 40 \\
\hline EVA & 54 & 52,9 & 58,3 & 66,7 & 51,9 & 50 & 57,1 & 60 \\
\hline Ns-Nc. & 7,9 & 3,9 & 25 & 11,1 & 7,4 & 12,5 & 4,8 & - \\
\hline $\begin{array}{l}\text { Registran el dolor } \\
\text { documentalmente }\end{array}$ & 23,9 & 22,2 & 30,8 & 44,4 & 20,7 & 24,2 & 26,1 & 18,2 \\
\hline $\begin{array}{l}\text { La institución } \\
\text { provee de escalas }\end{array}$ & 26,9 & 22,2 & 46,2 & 55,6 & 22,4 & 27,3 & 26,1 & 27,3 \\
\hline
\end{tabular}

Valores en porcentajes. UDA: unidad de dolor agudo; PID: programa institucional de dolor.

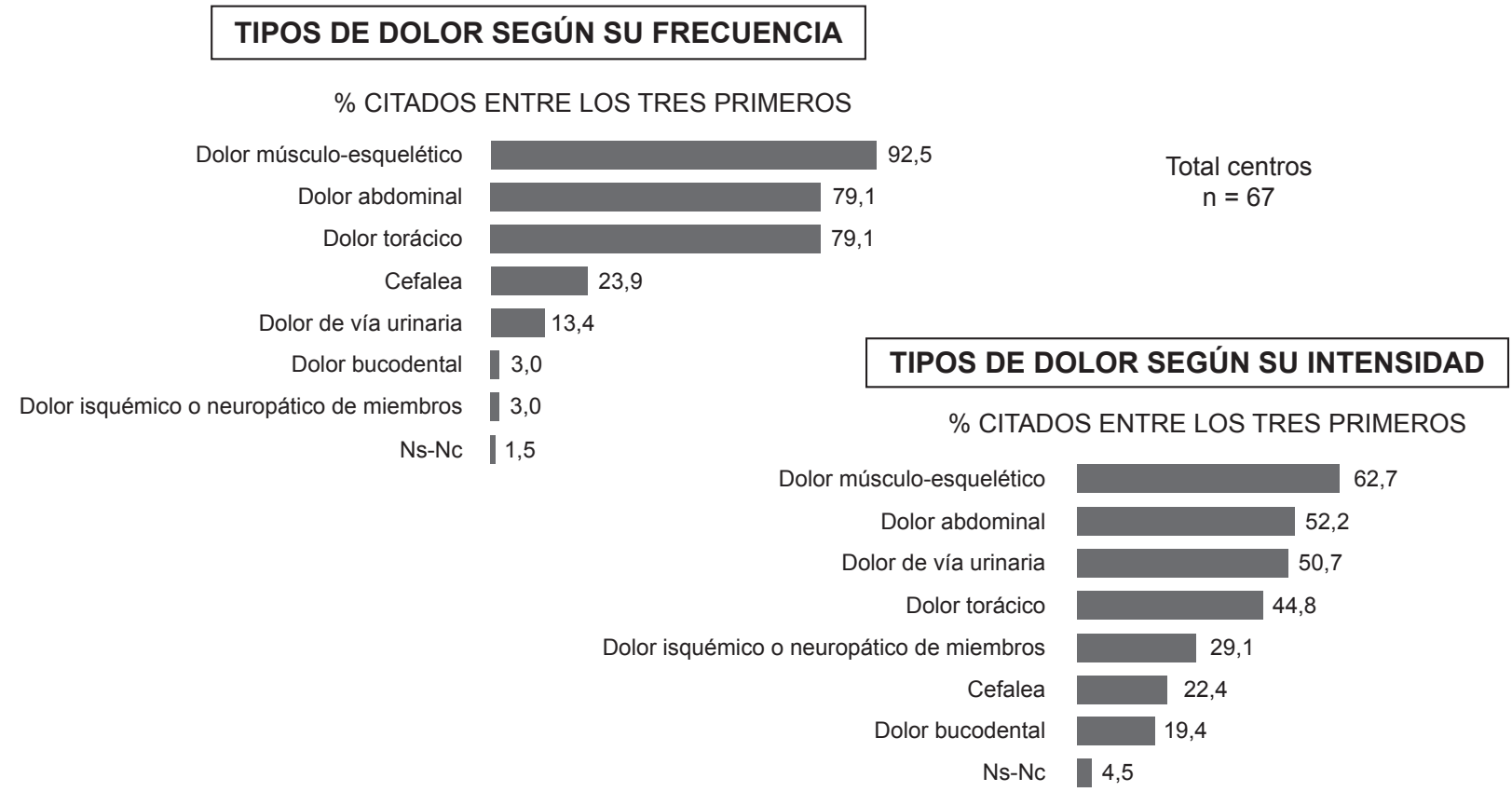

Fig. 3. Tipo e intensidad de los dolores más prevalentes.

Sólo 8 hospitales $(26,9 \%)$ facilitan a su personal escalas para medir el dolor, sobre todo en los hospitales con PIDUDA y en los no universitarios.

Para registrar el dolor como $5^{\text {a }}$ variable el $23,9 \%$ de los hospitales disponen de documentación específica impresa en papel o en registros informáticos en los $\mathrm{SU}$, con mayor porcentaje en aquellos con PID o UDA (44,4 \%) frente al $20,7 \%$ en los que no tienen UDA. Los protocolos también son más frecuentes en estos hospitales $(77,8 \%$ frente a $41,4 \%$ ) (Tabla III). 
TABLA III. ASPECTOS RELACIONADOS CON LA ADMINISTRACIÓN DE ANALGESIA EN DIFERENTES TIPOS DE HOSPITALES

\begin{tabular}{|c|c|c|c|c|c|c|c|}
\hline \multirow[b]{2}{*}{$\begin{array}{c}\text { Total } \\
(n=67)\end{array}$} & \multicolumn{2}{|c|}{ Tipo de formación } & \multicolumn{2}{|c|}{ UDA o PID } & \multicolumn{3}{|c|}{ Demanda atendida } \\
\hline & $\begin{array}{c}\text { Univers. } \\
\text { postgrado } \\
(n=54)\end{array}$ & $\begin{array}{c}\text { No } \\
\text { universi. } \\
(n=13)\end{array}$ & $\begin{array}{c}S i ́ \\
(n=9)\end{array}$ & $\begin{array}{c}N o \\
(n=58)\end{array}$ & $\begin{array}{c}\text { Menos de } \\
200 \text { pac. } \\
\text { día } \\
(n=33)\end{array}$ & $\begin{array}{c}\text { Entre } 200- \\
400 \text { pac. } \\
\text { día } \\
(n=23)\end{array}$ & $\begin{array}{c}\text { Más de } \\
400 \text { pac. } \\
\text { día } \\
(n=11)\end{array}$ \\
\hline
\end{tabular}

\begin{tabular}{lcccccccc}
\hline $\begin{array}{l}\text { Existen protocolos } \\
\text { analgésicos } \% \text { de } \\
\text { hospitales) }\end{array}$ & 46,3 & 46,3 & 46,2 & 77,8 & 41,4 & 36,4 & 60,9 & 45,5 \\
$\begin{array}{l}\text { Uso de opioides } \\
\text { mayores } \% \\
\text { pacientes con dolor) }\end{array}$ & 16,93 & 16,74 & 17,69 & 24 & 15,8 & 14,8 & 16,57 & 23,91 \\
$\begin{array}{l}\text { Uso de opioides } \\
\text { menores (\% } \\
\text { pacientes con dolor) }\end{array}$ & 25,42 & 26,93 & 19,15 & 26,33 & 25,3 & 23,88 & 26,74 & 27,27 \\
$\begin{array}{l}\text { Uso de paracetamol } \\
\text { AINE (\% pacientes } \\
\text { con dolor) }\end{array}$ & 72,28 & 73,56 & 67 & 76,56 & 71,6 & 69,36 & 76,7 & 71,82 \\
$\begin{array}{l}\text { Uso de anestésicos } \\
\text { locales } \% \text { pacientes } \\
\text { con dolor) }\end{array}$ & 8,45 & 8,87 & 6,69 & 9,78 & 8,24 & 5,24 & 12,57 & 9,45 \\
\hline
\end{tabular}

Valores en porcentajes. UDA: unidad de dolor agudo; PID: programa institucional de dolor.

\section{\% CITADOS ENTRE LOS TRES PRIMEROS}

\section{ANALGÉSICOS NO OPIOIDES}

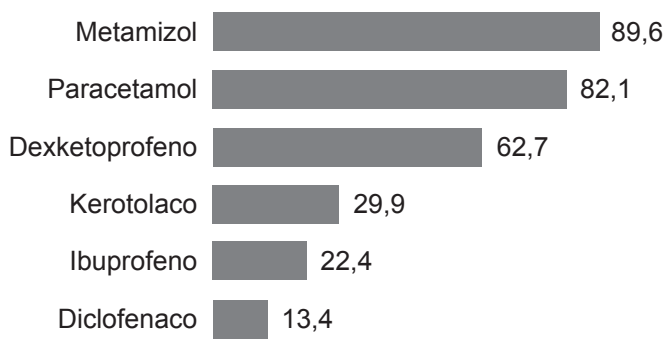

OPIOIDES

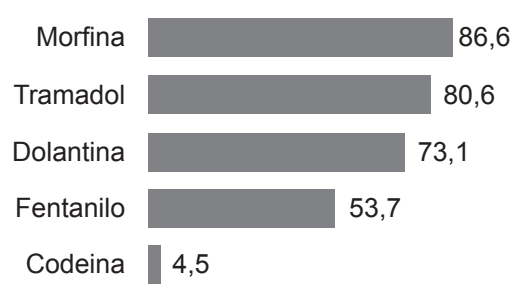

VÍA DE ADMINISTRACIÓN
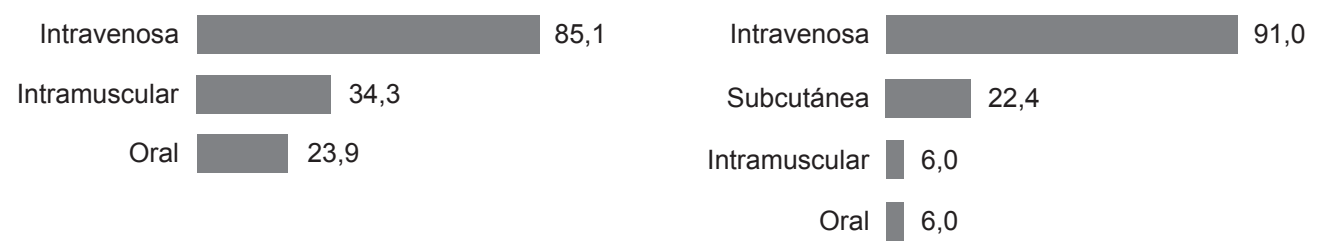

Fig. 4. Fármacos analgésicos más utilizados y vía de administración. 


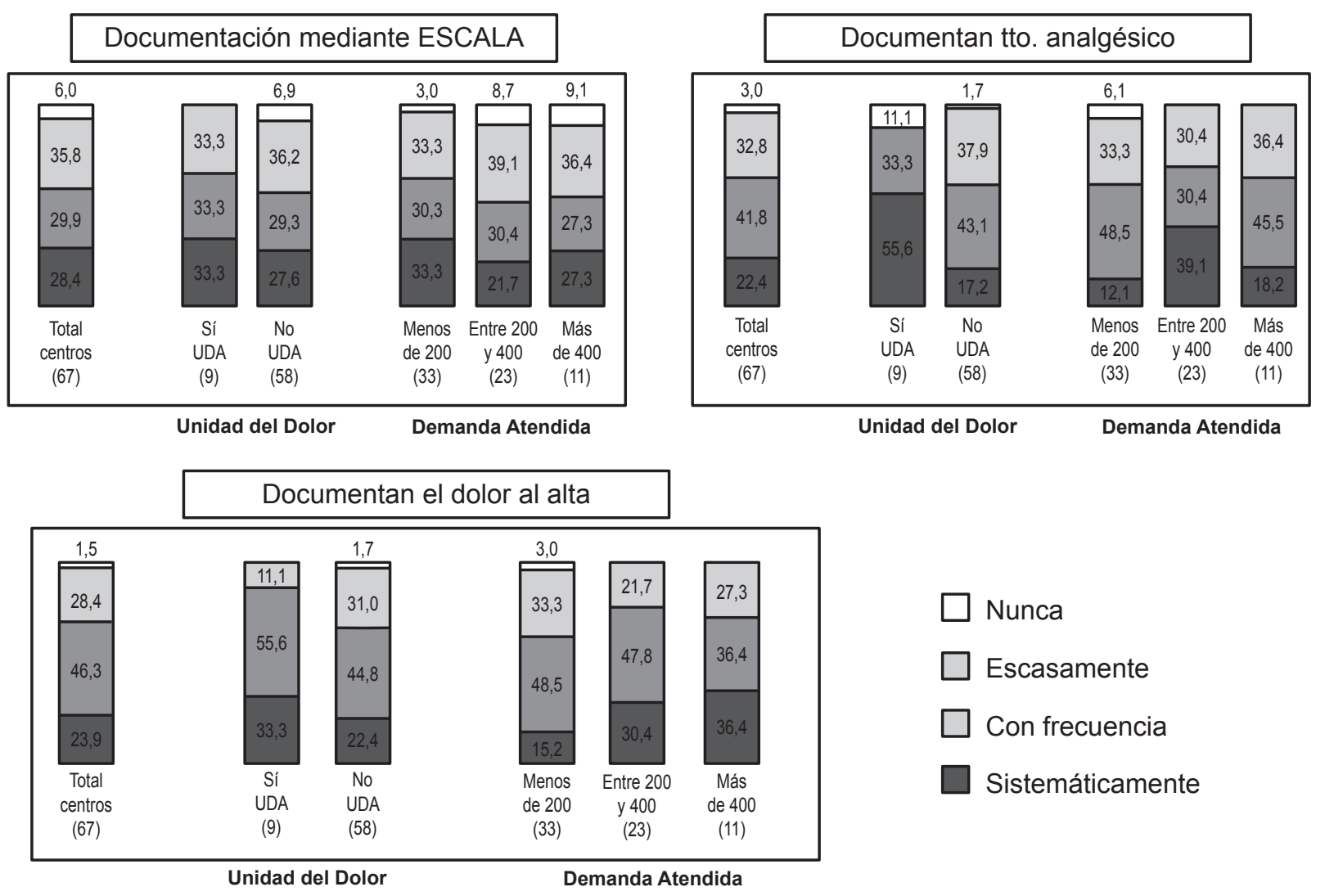

Fig. 5. Grado de documentación del dolor según diferentes tipos de hospitales. Valores en porcentajes.

Respecto a la formación del personal, sólo 14 hospitales $(20,9 \%)$ reconocieron tener programas o cursos específicos de dolor para el staff en los SU, acreditados en $42,9 \%$ de los casos. Para enfermería sólo tienen cursos los centros con PID-UDA y el 59,7 \% no tienen cursos de ningún tipo de formación en dolor. Cuando hay cursos para médicos en formación y enfermeras, sólo el 33,3 \% están acreditados (Tabla IV).

\section{DISCUSIÓN}

Con el muestreo realizado para este estudio hemos querido abarcar una información globalizada y representativa sobre determinados aspectos clínico-epidemiológicos y del manejo del dolor en los SU de los hospitales españoles, ya que incluía al $45 \%$ de todos aquellos con más de 400 camas. Con respecto a la estratificación de los centros, como era previsible, se produjo una correlación positiva entre el número de camas hospitalarias y el número de urgencias atendidas al día.

Sin haber encontrado ningún artículo que valore el dolor y su gestión mediante encuesta a los responsables médicos de la urgencia, la mayoría de los estudios sobre la gestión del dolor en los SU se basan en las encuestas a los pacientes y auditorías. Una encuesta como esta, aunque proporcione datos o percepciones de profesionales que podrían no coincidir exactamente con los que hubiera aportado el paciente o su historia clínica, ofrecería sin embargo una información nueva y pensamos que también valiosa.

Coincidiendo con las publicaciones europeas y americanas, en este estudio se pone de manifiesto, una vez más, que el dolor es un motivo frecuente de admisión en los SU hospitalarios, sin que las características de los centros influyeran manifiestamente en su presentación. No obstante, la incidencia global reflejada del $42,8 \%$ es muy inferior a la publicada en otros estudios que utilizan la entrevista al paciente o auditoría, por lo cual creemos que puede estar infravalorada. Al igual se podría intuir con la estimación del 15,9 \% para los pacientes que presentaban dolor de intensidad grave, cuando la mayoría de estudios revisados sobre el tema superan esta cifra.

$\mathrm{Al}$ igual que se refiere en otras publicaciones, el traumatismo es la causa más frecuente de este dolor, siendo el de origen músculo-esquelético el más prevalente y reconocido como de mayor intensidad, seguido del dolor abdominal 
TABLA IV. ASPECTOS RELACIONADOS CON LA FORMACIÓN ESPECÍFICA EN DOLOR DE LOS PROFESIONALES

\begin{tabular}{|c|c|c|c|c|c|c|c|c|}
\hline & \multirow[b]{2}{*}{$\begin{array}{c}\text { Total } \\
(n=67)\end{array}$} & \multicolumn{2}{|c|}{ Tipo de formación } & \multicolumn{2}{|c|}{$U D A$ o PID } & \multicolumn{3}{|c|}{ Demanda atendida } \\
\hline & & $\begin{array}{c}\text { Univers. } \\
\text { postgrado } \\
(n=54)\end{array}$ & $\begin{array}{c}\text { No } \\
\text { universi. } \\
(n=13)\end{array}$ & $\begin{array}{c}S i \\
(n=9)\end{array}$ & $\begin{array}{c}N o \\
(n=58)\end{array}$ & $\begin{array}{c}\text { Menos de } \\
200 \text { pac./dia } \\
(n=33)\end{array}$ & $\begin{array}{c}\text { Entre } 200- \\
400 \text { pac./ } \\
\text { dia }(n=23)\end{array}$ & $\begin{array}{c}\text { Más de } 400 \\
\text { pac./día } \\
(n=11)\end{array}$ \\
\hline \multicolumn{9}{|c|}{ Existe formación } \\
\hline $\begin{array}{l}\text { Sí, para staff } \\
\text { acreditada }\end{array}$ & $\begin{array}{c}14(20,9) \\
6(42,9)\end{array}$ & $\begin{array}{c}12(22,2) \\
6(50)\end{array}$ & $\begin{array}{c}2(15,4) \\
0(0)\end{array}$ & $\begin{array}{l}3(33,3) \\
1(33,3)\end{array}$ & $\begin{array}{c}11(19) \\
5(45,5)\end{array}$ & $\begin{array}{l}7(21,2) \\
3(42,9)\end{array}$ & $\begin{array}{c}3(13) \\
1(33,3)\end{array}$ & $\begin{array}{c}4(36,4) \\
2(50)\end{array}$ \\
\hline \multicolumn{9}{|c|}{ Existe formación } \\
\hline $\begin{array}{l}\text { Para } \\
\text { enfermería }\end{array}$ & $10(1,5)$ & $9(16,7)$ & $1(7,7)$ & $1(11,1)$ & - & $1(3)$ & - & - \\
\hline Para MIR & $17(25,4)$ & $15(27,8)$ & $2(15,4)$ & $2(22,2)$ & $15(25,9)$ & $7(21,2)$ & $7(30,4)$ & $3(27,3)$ \\
\hline $\begin{array}{l}\text { MIR y } \\
\text { enfermeras }\end{array}$ & $9(13,4)$ & $8(14,8)$ & $1(7,7)$ & $1(11,1)$ & $8(13,8)$ & $2(6,1)$ & $5(21,7)$ & $2(18,2)$ \\
\hline No existe & $40(59,7)$ & $31(57,4)$ & $9(69,2)$ & $5(55,6)$ & $35(60,3)$ & $23(69,7)$ & $11(47,8)$ & $6(54,5)$ \\
\hline
\end{tabular}

Valores en número (y porcentaje) de hospitales. UDA: unidad de dolor agudo; PID: programa institucional de dolor.

y del dolor torácico. Un aspecto que nos ha llamado la atención ha sido la frecuencia estimada de pacientes con dolor relacionada con la agudización de un proceso crónico (el 27,7\%), que contrasta con el $11 \%$ en algún estudio previo (21). Los pacientes con reagudizaciones de su dolor crónico suelen ser considerados en los SU como de "baja prioridad" complejos y especialmente problemáticos (22), cuya presencia en las urgencias hospitalarias implica para muchos ausencia de alternativas y falta de continuidad en su tratamiento. Este dato tan llamativo de la encuesta podría estar reflejando una realidad generalizada en nuestro país de falta de coordinación entre las unidades de dolor crónico y atención primaria (23).

El dolor es el síntoma tal vez más frecuentemente asociado a los cuadros que motivan la visita a urgencias y sólo se evalúa al ingreso de forma habitual en $58 \%$ de los hospitales, pero esta evaluación está poco documentada $(23,9 \%)$ y no se dan los recursos necesarios para ello (73\%). Si comparamos el dato de la documentación del dolor con los del estudio de Todd y cols. (7), un multicéntrico realizado en EE. UU. y Canadá que refiere un $83 \%$ de registro de la evaluación inicial del dolor, vemos que las diferencias son abrumadoras. Sin embargo, un dato positivo en nuestro caso es que la reevaluación tras la analgesia y al alta es incluso más elevada que al ingreso.

En este apartado de la evaluación del dolor parece existir una mayor concienciación en los hospitales con PID-UDA, donde se duplica su registro (igualmente parecen disponer de más recursos y protocolos analgésicos). Siendo la medida del dolor un derecho del paciente y una de las causas invocadas como más importantes para atenderlo de forma segura y eficaz $(24,25)$, entendemos que este debería ser un aspecto fundamental de mejora.

Aunque en este estudio no se ha evaluado el tiempo de demora de la analgesia, este registro, junto a la protocolización de la analgesia y la presencia de una enfermera en la admisión puede mejorar la gestión del dolor (26). La protocolización analgésica basada en el ascensor analgésico y la frecuente reevaluación del dolor evitaría que pacientes sin tratar fueran dados de alta.

En cuanto a los fármacos administrados, como sucede en los países de nuestro entorno, los AINE y paracetamol son los más utilizados, con bajo uso de opioides mayores, aunque superan a los reflejados en otros estudios (9). Actitudes como esperar a tener un diagnóstico y la opiofobia parecen seguir estando presentes. Aunque en los hospitales más grandes y con PID-UDA se administran más frecuentemente opioides mayores, no hay grandes diferencias en la práctica analgésica entre los diferentes hospitales de nuestro estudio. Por otra parte, consideramos que las vías de administración de analgésicos son adecuadas, con poco uso de la vía intramuscular y, afortunadamente, bastante uso de la vía intravenosa.

Los anestésicos locales son utilizados en el 8,4\% de los pacientes con dolor en los SU, aunque no se haya investigado la forma de hacerlo. Mientras infiltraciones e instila- 
ciones son aplicaciones sencillas y habituales, los bloqueos nerviosos periféricos requieren de entrenamiento especial y conocimiento de las técnicas anestésicas locorregionales, pero pueden tener sitio en el tratamiento del dolor agudo en las urgencias (27). Uno de los más utilizados con buenos resultados en las urgencias es el bloqueo femoral en fracturas de cadera (28).

La formación de los profesionales es otro punto importante y con repercusión en el manejo del dolor (16), tanto para médicos como para enfermeras, y por los datos aportados en la encuesta vemos que es deficitaria. Concienciar sobre la importancia de tratar el dolor con los medios necesarios, en el tiempo más corto posible, ampliando el abanico terapéutico y la combinación de fármacos, debería mejorar la calidad asistencial en nuestro medio. En este sentido intervenciones para mejorar el proceso del triaje $(12,14)$ pueden también mejorar la gestión de dolor en los SU.

Se ha percibido que los hospitales mayores, con más presión asistencial, parecen tener menos institucionalizado el manejo del dolor agudo postoperatorio (29) y pudiera ser que razones parecidas para que esto sea así tuvieran algo que ver con la falta de soluciones organizativas también en el manejo del dolor agudo en los SU. En cualquier caso, la presión asistencial se ha considerado que es un factor que puede influir negativamente en el adecuado control del dolor $(9,12)$.

En relación con los aspectos anteriormente mencionados, la implementación de unidades o programas específicos para el abordaje del dolor agudo podrían inducir mejoras visibles en su tratamiento. Sin embargo, estas organizaciones hospitalarias con proyección hacia los SU, desafortunadamente no abundan en los hospitales españoles, ya que por los resultados de la encuesta sólo un 13,4\% dispone de ellas, frente al $69,4 \%$ reflejado en una reciente auditoria australiana (30).

A pesar de las limitaciones de este estudio derivadas de su carácter de encuesta voluntaria y de que nos informa de tendencias sin poder hacer inferencias estadísticas, creemos que los datos aportados por los responsables médicos pueden ayudar a tener una visión global de la gestión del dolor en las urgencias hospitalarias de nuestro país, de los recursos que existen y de los campos de mejora en este ámbito.

\section{CONCLUSIONES}

El dolor sigue estando infravalorado en los SU de nuestros hospitales. Aunque se refiere que se mide, pocos lo registran y ponen medios para ello.

En un contexto con recursos limitados, los pocos hospitales con programa institucional para tratar el dolor en urgencias parecen más sensibilizados con su evaluación y tratamiento, así como más implicados en la formación de los profesionales, especialmente de la enfermería.
Por lo tanto, a nivel institucional e individual, tenemos un importante campo de mejora con la introducción obligatoria y documentada de sencillas escalas de dolor, protocolización de la analgesia asociada a la intensidad del mismo, implantación de estrategias que aceleren el inicio del protocolo analgésico, así como formación específica para médicos y enfermería.

\section{AGRADECIMIENTOS}

Agradecemos a la Sociedad Española de Medicina de Urgencias y Emergencias (SEMES) y a la Sociedad Española de Dolor (SED) por su patrocinio y colaboración, así como a Demométrica y Antonio Bustamante por su apoyo técnico y logístico.

\author{
CORRESPONDENCIA: \\ Fernando Caba Barrientos \\ Servicio de Anestesiología, Reanimación y Tratamiento del Dolor \\ Hospital Universitario de Valme \\ Avda. Bellavista, s/n \\ 41014 Sevilla \\ e-mail: fcababarrientos@terra.com
}

\section{BIBLIOGRAFÍA}

1. Wilson J, Pendelton J. Oligoanalgesia in the emergency department. Am J Emerg Med 1989;7:620-3.

2. Ducharme J, Barber C. A prospective blinded study on emergency pain assesment and therapy. J Emerg Med 1995;13:571-5.

3. Jhonston CC, Gagnon AJ, Fullerton L, Common C, Ladores M, Forlini S. One-week survey of pain intensity on admission to and discharge from the emergency department: A pilot study. J Emerg Med 1998;16(3):377-82.

4. Tanabe P, Buschmann M. A prospective study of ED pain management and the patient's perspective. J Emerg Nurs 1999;25(3):171-7.

5. Guru V, Dubinsky I. The patient vs caregiver perception of acute pain in the emergency department. J Emerg Med 2000;18(1):7-12.

6. Rupp T, Delaney KA. Inadequate analgesia in emergency medicine. Ann Emerg Med 2004;43:494-503.

7. Todd KH, Ducharme J, Choiniere M, Crandall CS, Fosnocht DE, Homel P, et al. Pain in the emergency department: Results of the Pain and Emergency Medicine Initiative (PEMI) multicenter study. J Pain 2007;8:460-6.

8. Tcherny-Lessenot S, Karwowski-Soulié F, Lamarche-Vadel A, Ginsburg Ch, Brunet F, Vidal-Trecan G. Management and relief of pain in a emergency department from the adults patients' perspective. J Pain Symptom Manag 2003;25:539-46.

9. Guéant S, Taleb A, Borel-Kühner J, Cauterman M, Raphael M, Nathan G, et al. Quality of pain management in the emergency department: Results of a multicentre prospective study. Eur J Anaesthesiol 2011;28(2):97-105. 
10. Gaakeer MI, Veugelers R, Houser CM, Berben SA, Bierens JJ. Acute pain at the emergency department: Better treatment required. Ned Tijdschr Geneseskd 2011;155:A2241.

11. Martin JS, Spiring R. Pain prevalence and patient preferences concerning pain management in the emergency department. Pflege 2006;19(6):326-34.

12. Ducharme J, Tanabe P, Homel P, Miner JR, Chang AK, Lee $\mathrm{J}$, et al. The influence of triage systems and triage scores on timeliness of ED analgesic administration. Am J Emerg Med 2008;26:867-73 .

13. Decosterd I, Hugli O, Tamches E, Blanc C, Mouhsine E, Givel JC, et al. Oligoanalgesia in the emergency department: Short-term beneficial effects on an education program on acute pain. Ann Emerg Med 2007;50:462-71.

14. Vazinai J, Knott JC. Mandatory pain scoring at triage reduces time to analgesia. Ann Emerg Med 2012;59:134-8.

15. Silka MA, Rott MM, Moreno G, Merrill L, Geiderman JM. Pain scores improve analgesic administration patterns for trauma patients in the emergence department. Acad Emerg Med 2004;11(3):264-70.

16. Yanuka M, Soffer D, Halpern P. An interventional study to improve the quality of analgesia in the emergency department. CJEM 2008;10(5):435-9.

17. Grupo de Dolor Agudo. Guía de dolor agudo postoperatorio. Muñoz y Ramón JM, coordinador. Madrid: Editorial de la SED; 2011.

18. Sociedad Española del Dolor. Valoración epidemiológica del Dolor en Urgencias. Barcelona: Boehringer Ingelheim. Europharma; 2000.

19. García-Castrillo Riesgo L, Gómez MA, Puig SR, González RJ, Peters ES, García Cases C. El dolor en procesos traumáticos agudos leves. Emergencias 2006;18:19-27.

20. Casal Codesido JM, Vázquez Lima J. Abordaje del dolor musculoesquelético en urgencias. Emergencias 2012;24:59-95.

21. Cordell WH, Keene KK, Giles BK, Jhones JB, Jhones JH, Brizendine EJ. The high prevalence of pain in emergency medical care. Am J Emerg Med 2002;20(3);165-9.

22. Wilsey BL, Fishman SM, Ogden C, Tsodikov A, Bertakis KD. Chronic pain management in the emergency department: A survey of attitudes and beliefs. Pain Medicine 2008;9(8):1073-80.

23. García Martínez F, Herrera Silva J, Aguilar Luque J. Tratamiento del dolor crónico en atención primaria. Rev Soc Esp Dolor 2000; 7:453-9.

24. Gordon DB, Rees SM, McCausland MR, Pellino TA, Sandford-Ring S, Smith-Helmenstine J, et al. Improving reassesment and documentation of pain management. Jt Comm J Qual Patient Saf 2008;34(9):509-17.

25. Unidad de Tratamiento del dolor. Estándares y recomendaciones de calidad y seguridad. Ministerio de Sanidad, Política Social e Igualdad; 2011.

26. Foschnot DE, Swanson ER. Use of a triage pain protocol in the ED. Am J Emerg Med 2007;25:791-3.

27. Crystal CS, Blankenship RB. Local anesthetics and peripheral nerve blocks in the emergency department. Emerg Med Clin North Am 2005;23(2):477-502.

28. Beaudoin FL, Nagdev A, Mrrchant RC, Becker BM. Ultrasound-guied femoral nerve blocks in elderly patients with hip fractures. Am J Emerg Med 2010;28:76-81.

29. Torres LM, Aguilar JL. Organización del tratamiento del dolor postoperatorio. Rev Esp Anestesiol Reanim 2010;57:199-200.

30. Fry M, Bennetts S, Huckson S. An Australian audit of ED pain management patterns. J Emerg Nurs 2011; 37:26974. 


\section{ANEXO 1. \\ Encuesta estructurada sobre el dolor en los servicios de urgencias}

\section{Clasificación de centros}

1. $\mathrm{N}^{\mathrm{o}}$ de camas de hospitalización

口 $>1.000, \quad \square$ entre 1.000 y $400, \quad \square$ entre 400 y $200, \quad \square<200$

2. Promedio de admisiones/día

$\square>400, \square$ entre 400 y $200, \quad \square<200$

3. Formación postgrado (al menos MIR de medicina de familia)

घí, $\square$ No

4. Unidad de dolor agudo o programa institucional de dolor agudo

口 Sí $\quad$ No

5. Personal sanitario del servicio de urgencias incluido en esa UDA o programa institucional

口 Sí a No

\section{Admisión y diagnóstico}

6. En un día normal, de cada 100 pacientes que ingresan en urgencias ¿en cuántos de ellos el dolor es el único o principal motivo de la admisión?: $\%$

7. Hablando única y exclusivamente de estos pacientes ¿qué porcentaje presenta ...?:

Agudización de dolor crónico:

Dolor asociado a traumatismos:

Dolor de intensidad grave:

$-\%$
$-\%$

8. Ordene los siguientes diagnósticos de dolor que se pueden presentar en urgencias según su frecuencia (1 el más frecuente, y sucesivos)

( ) Dolor músculo-esquelético

( ) Dolor abdominal

( ) Dolor torácico

( ) Dolor de la vía urinaria

( ) Cefalea

( ) Dolor buco-dental

( ) Dolor isquémico o neuropático de miembros

( ) Otros

9. Ordénelos según intensidad (1 para el de mayor intensidad, y sucesivos)

( ) Dolor músculo-esquelético

( ) Dolor abdominal

( ) Dolor torácico

( ) Dolor de la vía urinaria

( ) Cefalea

( ) Dolor buco-dental

( ) Dolor isquémico o neuropático de miembros

( ) Otros 


\section{Evaluación del dolor}

10. ¿Se evalúa y documenta en su centro el dolor con alguna escala?
○unca,
¿ Escasamente,
Con frecuencia,
๑ Sistemáticamente

11. ¿Se evalúa y documenta el resultado del tratamiento analgésico?

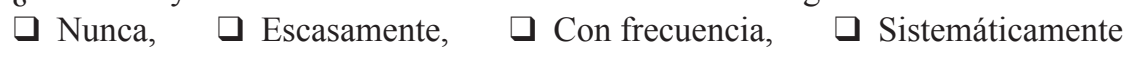

12. ¿Se evalúa y documenta el dolor al alta?

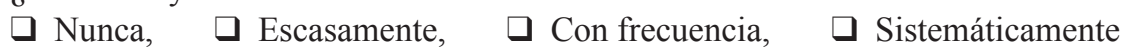

\section{Registro del dolor}

13. ¿La institución provee de escalas para la evaluación del dolor?

口í a No

14. ¿Existe entre la documentación de urgencias, impresa o informática, algún espacio destinado a registrar el dolor como quinta constante?

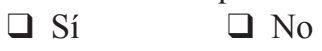

\section{Tratamiento}

15. ¿Existen protocolos para el tratamiento del dolor agudo en Urgencias?

¿ Sí

ㄱo

16. ¿En qué porcentaje de pacientes con dolor se utilizan los siguientes fármacos/técnicas analgésicas?

Opioides mayores: _ - \%

Opioides débiles: $---\%$

Paracetamol/AINE: $-{ }_{-} \%$

Infiltraciones, instilaciones o bloqueos con anestésicos locales: __ $\%$

17. Señale qué opioides son los más utilizados en urgencias para el tratamiento del dolor agudo en orden de frecuencia (1 el más utilizado, y sucesivos)
( ) Morfina
( ) Dolantina
( ) Fentanilo
( ) Metadona
( ) Tramadol
( ) Codeína

18. ¿Cuál es la vía más utilizada en urgencias para la administración de opioides?
○ Oral,
$\square$ Intramuscular,
๑ Subcutánea,
Intravenosa

19. Señale qué analgésicos no opioides son los más utilizados en urgencias para el tratamiento del dolor agudo (1 el más utilizado, y sucesivos)

( ) Paracetamol

( ) Metamizol

( ) Dexketoprofeno

( ) Ketorolaco

( ) Diclofenaco

( ) Ibuprofeno

( ) Otro 
20. ¿Qué vía es la más utilizada en urgencias para la administración de analgésicos no opioides?

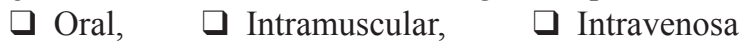

\section{Formación}

21. ¿Existen programas o cursos de formación específica en dolor para el personal del staff de urgencias? 口í $\square$ No

22. Si la anterior respuesta es afirmativa, ¿están acreditados?

口 Sí a No

23. ¿Existen cursos de formación en dolor para enfermería o MIR que inician, o van a iniciar, actividad asistencial en urgencias?

口 Sí a No

24. Si la anterior respuesta es afirmativa, ¿están acreditados?
○í
$\square$ No 